\title{
The structure of concentrated aqueous solutions of chromium nitrate and cerium chloride studied by X-ray diffraction and Raman spectroscopy
}

\author{
M. Isabel Cabaço ${ }^{\text {a,b }}$, M.I. de Barros Marques ${ }^{\text {a,b }}$, A.M. Gaspar ${ }^{\text {a,c }}$, \\ M. Alves Marques ${ }^{a, b, *}$, M. Margarida Costa ${ }^{d}$ \\ ${ }^{a}$ Centro de Física Atómica, Universidade de Lisboa, Av. Prof. Gama Pinto2, 1649-003 Lisboa Codex, Portugal \\ ${ }^{\mathrm{b}}$ Departamento de Física, Instituto Superior Técnico, Universidade Técnica de Lisboa, Av. Rovisco Pais, 1049-001 Lisboa, Portugal \\ ${ }^{\mathrm{c}}$ Forschungsneutronenquelle Heinz Maier-Leibnitz, FRM II and Physics Department E13, Technische Universitaet Muenchen, \\ Lichtenbergsstrasse 1, 85748 Garching, Germany \\ ' Departamento de Física de Universidade de Coimbra, Apartado 3008, 3000 Coimbra, Portugal
}

Available online 24 August 2007

\begin{abstract}
Concentrated aqueous solutions of chromium nitrate and cerium chloride were investigated by X-ray diffraction and Raman spectroscopy. The X-ray diffraction patterns of two sets of solutions (1.60 up to $2.48 \mathrm{~mol} \mathrm{dm}^{-3}$ for chromium nitrate and 0.98 up to $2.81 \mathrm{~mol} \mathrm{dm}^{-3}$ for cerium chloride) display an intense maximum, prepeak, in the angular region about $Q_{0} \sim 0.9 \AA^{-1}(Q=4 \pi \sin \theta / \lambda, \theta$ being the diffracting angle). The value of $Q_{0}$ corresponding to these maxima is proportional to the power $1 / 3$ of the molar concentration. The proportionality coefficient has the value that should be observed for a close packing (fcc network) if assumed for cations. This study demonstrates, once more, the existence of an intermediate range order (up to $\sim 10 \AA$ ) in very concentrated aqueous solutions of salts constituted by small ions of different valences, range order previously reported by these authors in many articles. Raman experiments are in consonance with X-ray diffraction results.
\end{abstract}

(C) 2007 Elsevier B.V. All rights reserved.

Keywords: Intermediate range order; Chromium nitrate cerium chloride; Concentrated aqueous solutions

\section{Introduction}

The structure of liquid aqueous solutions has been investigated since more than a century. This subject is interesting from the point of view purely scientific and is important for the technologies concerning the life: preservation of the environment, food production, medical sciences, etc.

Some authors of this contribution have paid a continued attention to concentrated aqueous solutions of salts of metallic cations. When the salts are constituted by ions of different valences X-ray diffraction investigations demonstrate that an intermediate range order should exist in these solutions [1-13]. If the constituent ions are both monovalent, as for instance

\footnotetext{
* Corresponding author. Centro de Física Atómica da Universidade de Lisboa, Av.Prof. Gama Pinto, 2, 1649-003 Lisboa, Portugal. Tel.: +351 217904879; fax: +351 217954288 .

E-mail address: marques@cii.fc.ul.pt (M.A. Marques).
}

sodium chloride, or both divalent, as the magnesium or the zinc sulphate, no trace of an intermediate order was detected in the diffraction patterns. The concentration values of these investigated salts in these solutions are about $1-3 \mathrm{~mol} \mathrm{dm}^{-3}$. These studies followed researches of Prins [14] in this area.

Raman spectroscopy was used also in order to detect the existence of coordination shells around cations when these are the ions of higher valence in the solved salt [15-17].

In the X-ray diffraction patterns of a large number of these solutions a maximum of intensity, prepeak, appears at $Q_{0} \sim 0.9 \AA^{-1}(Q=4 \pi \sin \theta / \lambda, \theta$ the diffracting angle $), Q_{0}$ exhibiting a variation with the concentration $c$ in agreement with the law $Q_{0} \propto c^{1 / 3}$. The proportionality coefficient corresponds to an arrangement in close-packing (fcc lattice) of the ions of higher valence. The range of the adequacy of this intermediate range order is limited to about $10 \AA$.

The validity of this hypothesis for a large number of solutions at various concentrations has suggested to the authors the possibility of building molecular models for these liquids. As the 
design of the models depends on a number of arrangements and parameters (considering always the hypothesis of the existence of the close packing of the ions of largest valence) they can not lead to accurate quantitative conclusions but they permit to discuss important qualitative aspects.

The details of the molecular correlations between ions and between ions and water molecules are given in Section 3. But some general considerations may be advanced here. In the elaboration of their models the authors start to constitute around every ion of largest valence inside the aqueous solution a representative molecular cloud. This (discrete) cloud never exceeds the stoichiometric molecular ensemble that corresponds to that ion of largest valence. For obtaining an easy calculation of the X-ray intensity pattern of the molecular model a structure as symmetrical as possible was adopted for the unitary cell. In many aqueous solutions this architecture is successful $[2,6,7]$ but it does not seem possible in all the cases, for instance, for chromium nitrate aqueous solutions here referred. This impossibility does not correspond to the inexistence of the liquid close-packing but only to the inadequacy of the approximation of assuming an excessively symmetrical structure for the model.

Therefore it should be emphasized that the hypothesis of a liquid-type quasi-close-packing of the ions of larger valence can give a useful indication about the presence of counter-ions in the neighbourhood of the close-packed ions. Two examples:

i) X-ray diffraction patterns of two quasi-equimolecular solutions of aluminium, magnesium, yttrium, lanthanum chloride and bromide [1,5,7], for instance, show that the prepeak intensity, in the bromide solutions, is smaller than the intensity observed with the chloride solutions. This result is interpreted by the analysis of molecular models suggested by the authors: the halides are located, in chloride and bromide solutions corresponding to the same cation, outside the first coordination shell of the metallic cations. The existence of destructive interferences of the X-rays scattered by the anions in the angular region of the prepeak can explain this observed behaviour. Raman and EXAFS experiments confirmed that in the first coordination shell of lanthanum and yttrium cations only water molecules are included $[5,7]$. Previous Raman investigations of the structure of solutions of aluminium or magnesium salts had demonstrated that no anion is present in the solvation shells of these cations [15].

ii) When X-ray patterns of solutions of indium chloride are compared with patterns of quasi equimolecular solutions of indium bromide $[2,6]$ the prepeak is clearly more intense in the bromide solution pattern. It was possible to interpret this experimental result by assuming that in the molecular model two halide anions are included in the solvation shell of the indium cation. This hypothesis was corroborated by EXAFS studies implemented afterwards $[18,19]$. Comparison between the aqueous solutions are appropriate because approximately the same value is attributed to the ionic radii of the chloride and bromide anions. So the equimolecular chloride and bromide solutions may be considered as practically isomorphous.

Another example concerns a qualitative intervention of the molecular models when the X-ray diffraction patterns of aqueous solutions of lanthanum or aluminium chloride at different temperatures are compared [20]. It is shown that the small maxima observed for lanthanum at $\sim 1.7 \AA^{-1}$ (Fig. 1) and a demarcation of two maxima at $\sim 2.5 \AA^{-1}$ for aluminium chloride solutions (Fig. 2) practically disappear at $425 \mathrm{~K}$. A summarizing analysis of the molecular models suggested to interpret the profile of the pattern intensity of both solutions seems to demonstrate that the correlations where anions play an important role are the most affected by the modification of the temperature value. Moreover, the intensity and the profile of the prepeak are not much modified when the temperature of the solution rises. Also the maxima corresponding to correlations between the cation and its hydration water molecules are not strongly changed by altering the temperature of the solution.

The conclusion about the existence of an intermediate range order founded on the presence of a prepeak in the X-ray and neutron scattering patterns of soft matter (liquids, glasses...) has been analyzed and discussed, by a number of scientists [14,2154]. Systematic molecular dynamics simulations of aqueous solutions of salts constituted by trivalent cations combined with monovalent anions, that fully corroborate our fundamental assumption of the quasi-close-packing model, were recently implemented by Ribeiro [55]. In a second article [56] the same author interprets also satisfactorily the collective dynamics of a $2.8 \mathrm{~mol} \mathrm{dm}^{-3}$ aqueous solution of $\mathrm{EuCl}_{3}$ by the hypothesis of the liquid-type quasi-close-packing (LTQCP).

In this contribution results obtained for chromium nitrate and cerium chloride solutions are discussed. Aqueous solutions of the salts here mentioned have been discussed by several authors $[26,27,56-70]$.

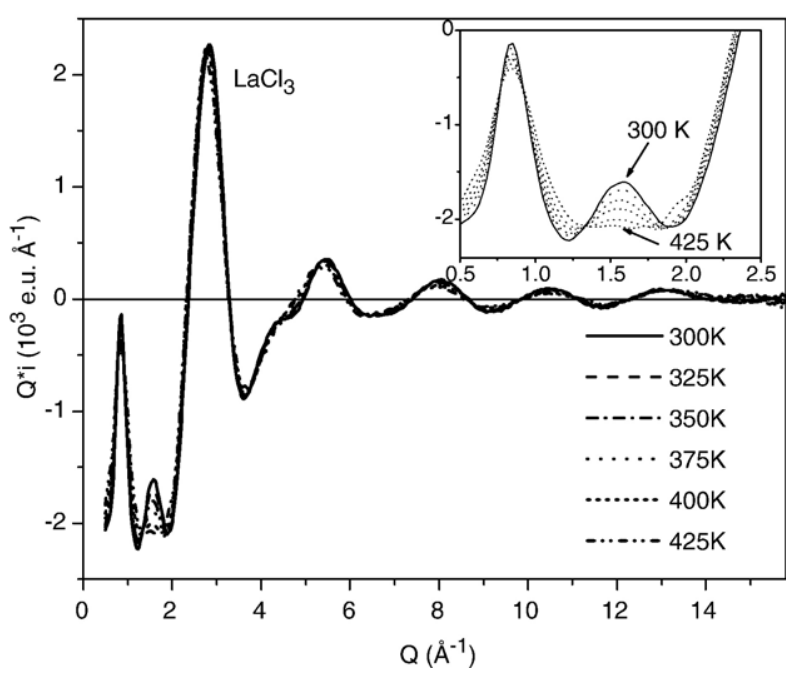

Fig. 1. Evolution with the temperature of the profile of the X-ray diffraction reduced intensity of the $3.03 \mathrm{~mol} \mathrm{dm}^{-3} \mathrm{LaCl}_{3}$ aqueous solution from $300 \mathrm{~K}$ up to $425 \mathrm{~K}$ (detailed dependence in the relevant $Q$ range is presented in the inset). 


\section{Experiments and data reduction}

The aqueous solutions were prepared by weight, dissolving commercially available salts in bidistilled water. Hydrated $\mathrm{Cr}$ $\left(\mathrm{NO}_{3}\right)_{3}$, was obtained from Aldrich, $\mathrm{AlCl}_{3}, \mathrm{Cu}\left(\mathrm{NO}_{3}\right)_{2}$ and $\mathrm{CeCl}_{3}$ from Merck and $\mathrm{Ce}\left(\mathrm{NO}_{3}\right)_{3}$ and $\mathrm{La} \mathrm{Cl}_{3}$ from Fluka.

The composition of the aqueous solutions investigated was monitored by chemical analysis (see Table 1).

The aqueous solutions investigated by Raman spectroscopy were filtered through nitrocellulose membranes prepared in our laboratory in order to reduce the background in the Raman spectra due to impurities of the solution.

The X-ray diffraction patterns of the aqueous solutions were obtained at the European Synchrotron Radiation Facility (ESRF, Grenoble) on the high energy beamline ID15b using monochromatized radiation of high energy $(88.54 \mathrm{keV}$, $0.140 \AA$ ). The $2.48 \mathrm{~mol} \mathrm{dm}^{-3} \mathrm{Cr}\left(\mathrm{NO}_{3}\right)_{3}$ solution was also measured in a conventional set-up in our laboratory (CFAUL, Lisbon).

At ESRF, the vacuum chamber was sealed with mylar windows $(100 \mu \mathrm{m})$ for the incident and scattered X-rays paths. The detection system was an MAR online image plate scanner $(2300 \times 2300$ pixels, pixel size $0.15 \mathrm{~mm})$. The one-dimensional diffraction patterns were obtained by integration of the diffraction rings of the $2 \mathrm{D}$ patterns. In order to gather information over the relevant $Q$ domain the X-ray diffraction patterns were acquired at two different distances: $320 \mathrm{~mm}$ and $960 \mathrm{~mm}$ from the sample to the detector. A tube filled with helium was used to reduce significantly the contribution from air scattering to pattern intensity at the longest path between the sample and the detector.

In the conventional set-up, $\mathrm{RhK} \alpha$ radiation $(\lambda=0.615 \AA)$ was monochromatized by reflection from a lithium fluoride crystal. The intensities were measured on a Philips $\theta-2 \theta$ horizontal goniometer, slightly modified, with a scintillator counter as detector. The range of the scattering angle allows to obtain

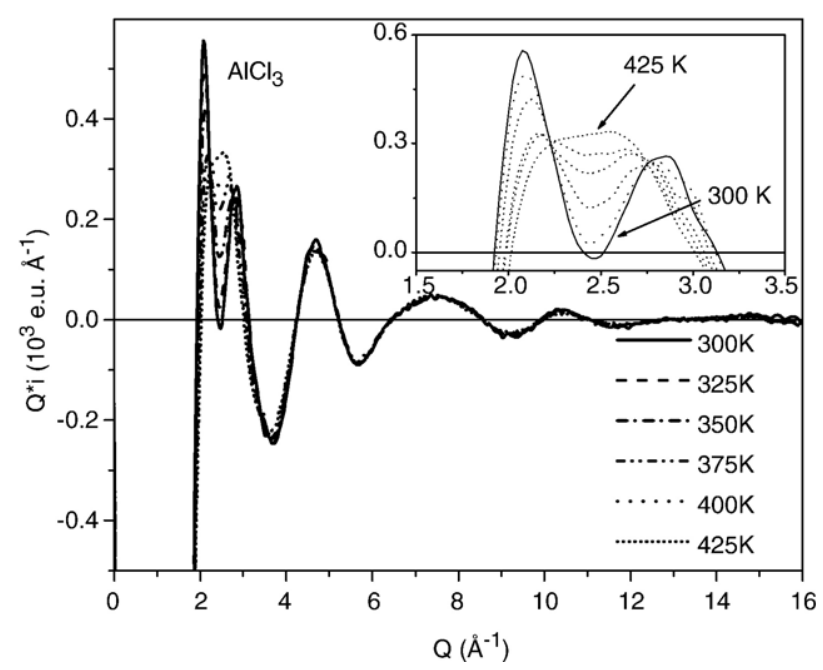

Fig. 2. Evolution with the temperature of the profile of the X-ray diffraction reduced intensity of the $2.86 \mathrm{~mol} \mathrm{dm}{ }^{-3} \mathrm{AlCl}_{3}$ aqueous solution from $300 \mathrm{~K}$ up to $425 \mathrm{~K}$ (detailed dependence in the relevant $Q$ range is presented in the inset).
Table 1

Concentration and density of the aqueous solutions investigated; $n$ being the calculated number of water molecules per cation

\begin{tabular}{lrll}
\hline Aqueous solution & \multicolumn{1}{l}{$n$} & $d_{20}{ }^{\circ} \mathrm{C}\left(\mathrm{g} \mathrm{cm}^{-3}\right)$ & Concentration $\left(\mathrm{mol} \mathrm{dm}^{-3}\right)$ \\
\hline $\mathrm{LaCl}_{3}$ & 16.3 & 1.634 & 3.03 \\
$\mathrm{AlCl}_{3}$ & 18.0 & 1.309 & 2.86 \\
$\mathrm{Cr}\left(\mathrm{NO}_{3}\right)_{3}$ & 18.2 & 1.407 & 2.48 \\
& 24.7 & 1.347 & 1.97 \\
& 31.3 & 1.288 & 1.60 \\
$\mathrm{Cu}\left(\mathrm{NO}_{3}\right)_{3}$ & 9.5 & $1.712\left(22{ }^{\circ} \mathrm{C}\right)$ & 4.84 \\
$\mathrm{CeCl}$ & 17.8 & 1.593 & 2.81 \\
& 27.5 & 1.406 & 1.90 \\
& 55.1 & 1.212 & 0.98 \\
$\mathrm{Ce}\left(\mathrm{NO}_{3}\right)_{3}$ & 24.1 & 1.624 & 2.13 \\
& 50.8 & 1.322 & 1.07 \\
& 98.2 & 1.173 & 0.56 \\
& 196 & 1.090 & 0.28 \\
\hline
\end{tabular}

patterns up to a momentum transfer $Q=4 \pi \sin \theta / \lambda \sim 16 \AA^{-1}$. Several runs were accumulated with a constant number of counts at each position.

The X-ray diffraction patterns of the $\mathrm{LaCl}_{3}$ and $\mathrm{AlCl}_{3}$ solutions were obtained from room temperature up to $425 \mathrm{~K}$ and all the other solutions were studied only at room temperature. Plane parallel cells were used by transmission between kapton or nitrocellulose windows $(25 \mu \mathrm{m})$ with a thickness of $2 \mathrm{~mm}$ (for $\lambda=0.140 \AA$ ) or $0.7 \mathrm{~mm}$ (for $\lambda=0.615 \AA$ ).

Corrections were made for empty container, air (and helium) scattering contributions, absorption and geometry.

Finbak's method, as reformulated by Warren [71], was applied to the experimental data and used to calculate the following total pair-correlation function of the solution $g(r)$

$g(r)=1+\left(2 \pi^{2} r \rho_{\mathrm{e}} \sum_{\mathrm{uc}} Z_{j}\right)^{-1} \int_{0}^{Q_{\max }} Q i^{\prime}(Q) e^{-b Q^{2}} \sin (r Q) \mathrm{d} Q$

where $\rho_{\mathrm{e}}$ is the average electron density of the solution, $Z$ the atomic numbers and $I^{\prime}(Q)$ the reduced intensity defined from the total intensity $I_{\mathrm{eu}}(Q)$ as

$i^{\prime}(Q)=\left(I_{\mathrm{eu}}(Q)-\sum_{\mathrm{uc}} f_{j}^{2}\right) / F^{2}(Q)=i(Q) / F^{2}(Q)$

where $F(Q)=\sum_{\text {uc }} f_{j} / \sum_{u c} Z_{j}$ is a sharpening factor. The units of composition uc are always referred to one cation in all solutions. For the atoms and the ions the coherent scattering factors $f_{j}$ were taken from X-ray tables [72] and corrected for anomalous scattering [73]. Incoherent scattered intensities corrected for the Breit-Dirac recoil factor were calculated from Palinkas and Radnai [74]. For water the coherent scattering factors and the incoherent scattered intensity from Hadju were used [75]. The corrected intensities were scaled to absolute units using the Krogh-Moe method [76,77].

Different values of $Q_{\max }$ were tested for both the Krogh-Moe method and the Fourier inversion. In the data analysis presented here we used $Q$ values up to $18 \AA^{-1}$ (ESRF) and up to $14 \AA^{-1}$ (CFAUL). For these values of $Q, b$ was set equal, respectively, to $0.006 \AA^{2}$ and to $0.01 \AA^{2}$ in the modification function $e^{-b Q^{2}}$. 

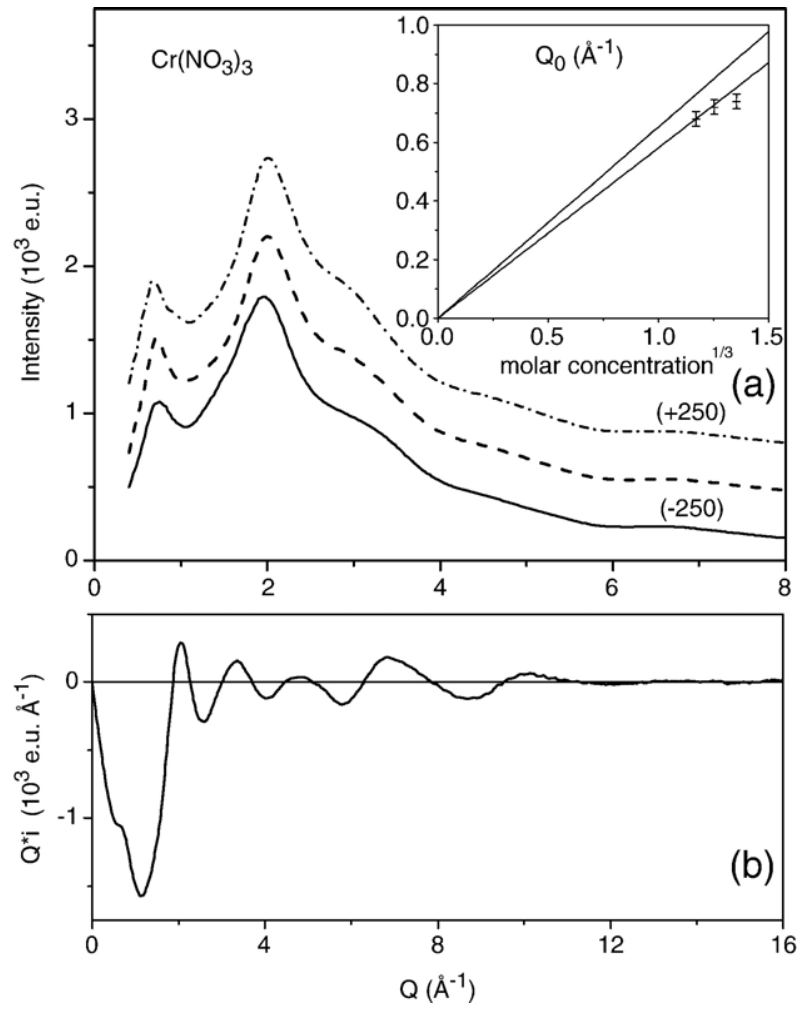

Fig. 3. (a) X-ray diffraction intensity of patterns corresponding to different concentrated $\mathrm{Cr}\left(\mathrm{NO}_{3}\right)_{3}$ aqueous solutions: (-) $2.48 \mathrm{~mol} \mathrm{dm}^{-3},(--) 1.97 \mathrm{~mol}$ $\mathrm{dm}^{-3}$ and $(-\cdot-) 1.60 \mathrm{~mol} \mathrm{dm}^{-3}$. In the inset the variation of the position of the prepeak versus the power $1 / 3$ of the molar concentration is represented. The lower straight line was calculated considering that the hydrated cations are locally ordered in a face-centered close-packing; the upper line fits a hypothetical arrangement (not verified by the experimental results) of the cations in a liquidtype quasi-simple-cubic-packing. b) Reduced intensity of the $2.48 \mathrm{~mol} \mathrm{dm}^{-3} \mathrm{Cr}$ $\left(\mathrm{NO}_{3}\right)_{3}$ aqueous solution.

The cut-off produces spurious peaks and decreases the spatial resolution, but this is not relevant for distances larger than $1 \AA$. A final correction has been made to reduce the spurious oscillations in the pair-correlation function $g(r)$ at small distances (less than $1 \AA$ ). The so-called pair-correlation function $g(r)$

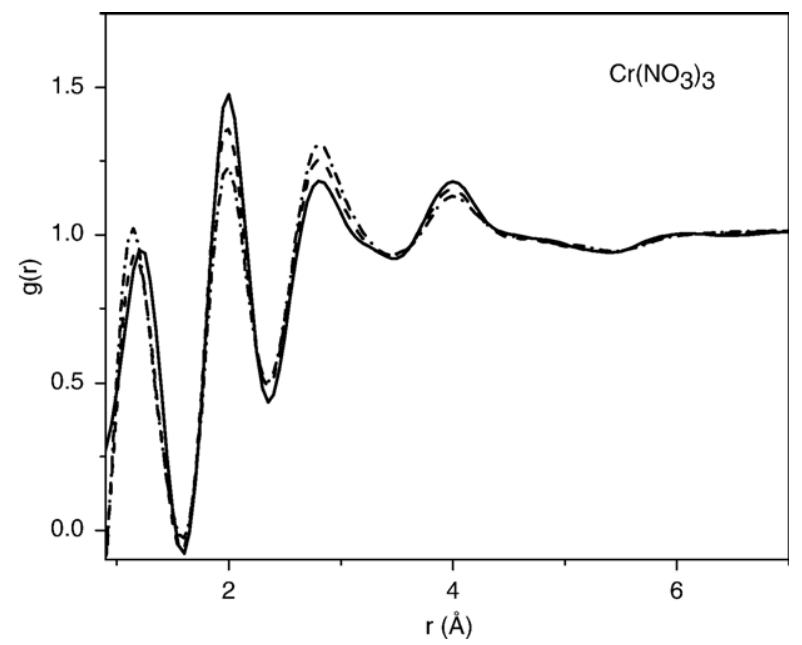

Fig. 4. Pair-correlation function $g(r)$ of different concentrated $\mathrm{Cr}\left(\mathrm{NO}_{3}\right)_{3}$ aqueous solutions: (-) $2.48 \mathrm{~mol} \mathrm{dm}^{-3},(--) 1.97 \mathrm{~mol} \mathrm{dm}^{-3}$ and (-•-) $1.60 \mathrm{~mol} \mathrm{dm}^{-3}$. was then back-transformed in order to obtain the corrected dependent intensity $i(Q)$.

Polarized $I_{\mathrm{VV}}$ and depolarized $I_{\mathrm{HV}}$ Raman spectra (except for $\mathrm{Cu}\left(\mathrm{NO}_{3}\right)_{3}$ solutions) were recorded on a Jobin-Yvon HR800 spectrometer at the Laboratoire de Physico-Chimie Moléculaire (LPCM), Université Bordeaux 1 (France) with Spectra Physics argon-ion and krypton-ion laser sources operating, respectively, at wavelengths of $514.5 \mathrm{~nm}$ and $752.5 \mathrm{~nm}$. Spectra were recorded using the back-scattering geometry with a resolution of $\sim 3 \mathrm{~cm}^{-1}$.

The Raman spectra of $\mathrm{Cu}\left(\mathrm{NO}_{3}\right)_{3}$ solutions were obtained on a Spex 1403 spectrometer, a Spectra Physics argon-ion laser operating at $488 \mathrm{~nm}$. The usual $90^{\circ}$ scattering geometry was used with a resolution of $\sim 15 \mathrm{~cm}^{-1}$.

\section{Molecular models}

We assume that the superposition of the X-rays scattered by the ions and molecules produces a total dependent intensity that may be computed by the Debye formula

$i(Q)=\sum_{i \neq k} f_{i} f_{k} \frac{\sin \left(Q r_{i k}\right)}{Q r_{i k}}$

$f_{i}$ being the atomic or molecular scattering factors, $r_{i k}$ the distances between the atoms or molecules $i$ and $k$. The method has already been described [13]. Two different internal configurations are considered in distinct regions: the first configuration, near an arbitrary central particle, is crystallinetype (discrete distribution); the other one, is simulated by a (random) uniform distribution. This uniform distribution that starts at a distance $r_{\text {th }}$ (threshold of this continuum) goes up to large distances and should simulate, plausibly, the space averaged structure of the solutions as it is "seen" by the arbitrary central particle at large distances in the bulk of the liquid. This continuum may not produce intensities larger than that one obtained from the discrete distribution in the $Q$ domain which is relevant for results interpretation $\left(Q>0.7 \AA^{-1}\right)$.

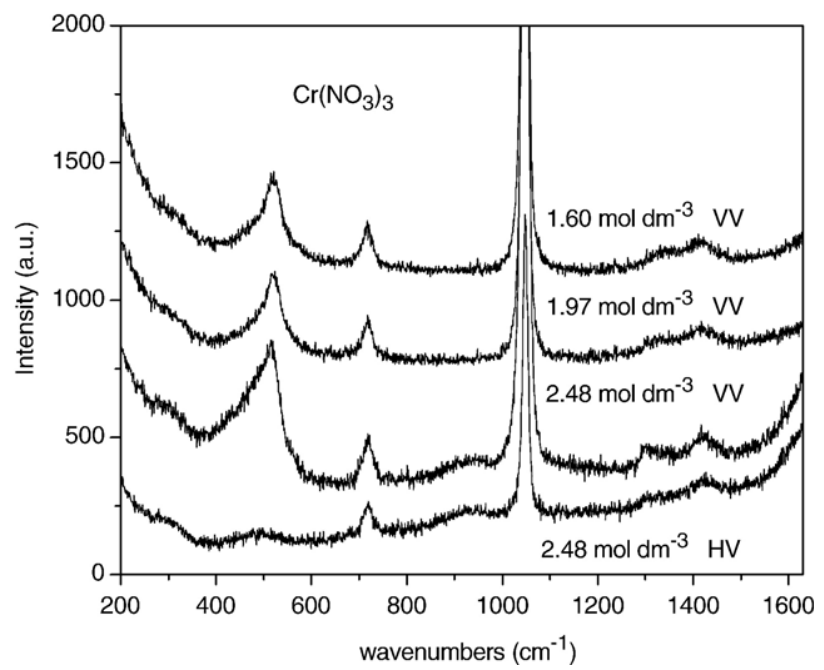

Fig. 5. Polarized $I_{\mathrm{Vv}}$ and depolarized $I_{\mathrm{HV}}$ Raman spectra of concentrated $\mathrm{Cr}\left(\mathrm{NO}_{3}\right)_{3}$ aqueous solutions. 


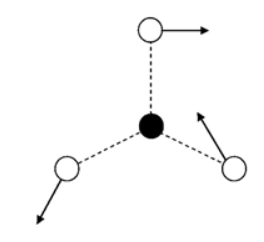

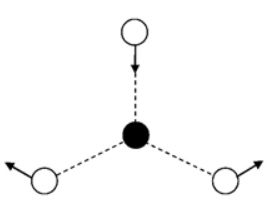

$v_{4}$

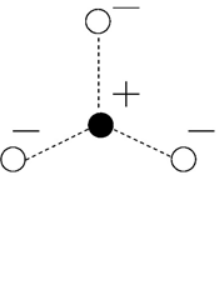

$v_{2}$
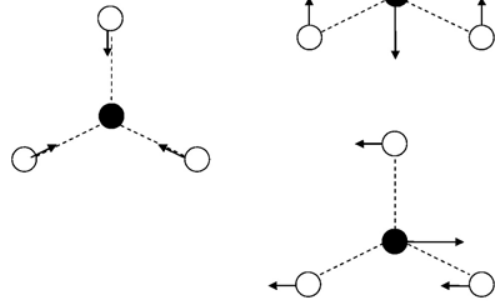

$v_{1}$

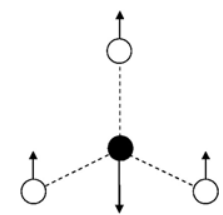

$v_{3}$

Fig. 6. Normal vibrations of the nitrate anion $(-\mathrm{N} ; \mathrm{O}-\mathrm{O})$ assumed as centered equilateral triangle. The vibrational mode $v_{2}$ is the only one inactive in the Raman spectrum. The modes $v_{3}$ and $v_{4}$ are double degenerated.

As for the discrete distribution, only a fraction of the shell of the first neighbours around the hydrated cations is taken into account, i.e. that one concerning those that face the central ion. It should be emphasized that the twelve neighbours, hydrated cations, of a central hydrated cation may not be located in the geometric sites of the face-centered cubic lattice. Indeed, the thermal energy at room temperature causes positional fluctuations around these geometric sites and only a liquid-type quasiclose packing should be representative [13].

Taking into account the electrostatic interactions and the sizes of the ions and of the water molecules for these concentrated solutions $\left(1-3 \mathrm{~mol} \mathrm{dm}^{-3}\right)$ we find that the anions are between two cations located in a region (approximately) equidistant from them.

\section{Results and interpretation}

In this article the authors suggest the existence in concentrated aqueous solutions of chromium nitrate (1.60 up to $2.48 \mathrm{~mol} \mathrm{dm}^{-3}$ ) of a liquid-type quasi-close-packing of the

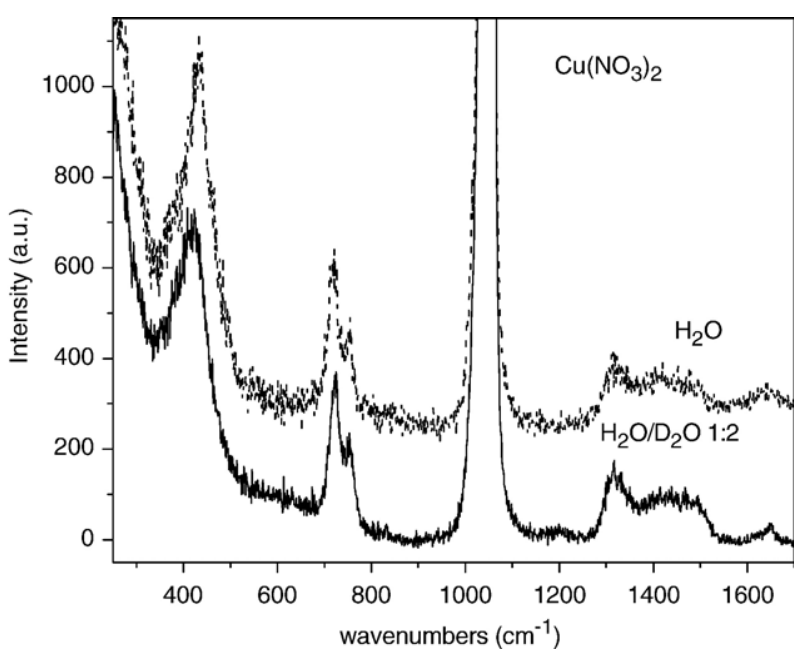

Fig. 7. Polarized $I_{\mathrm{VV}}$ Raman spectra of $4.84 \mathrm{~mol} \mathrm{dm}^{-3} \mathrm{Cu}\left(\mathrm{NO}_{3}\right)_{2}$ aqueous solution: in hydrogenated water (upper) and in a mixture of hydrogenated and deuterated water (lower) for which the molar fraction of hydrogenated water is $1 / 3$. cations. This hypothesis is based on the variation with the concentration of the solution of the low angle profile $\left(Q \sim 0.9 \AA^{-1}\right)$ in their X-ray diffraction patterns (Fig. 3a). If the intermediate range order were described by another arrangement, for instance, by a liquid-type quasi-simple-cubic-packing a law, $Q_{0} \propto c^{1 / 3}$ should be yet obtained but with a different proportionality coefficient. In Fig. 3a the two straight lines corresponding to the two types of distribution are displayed in the inset.

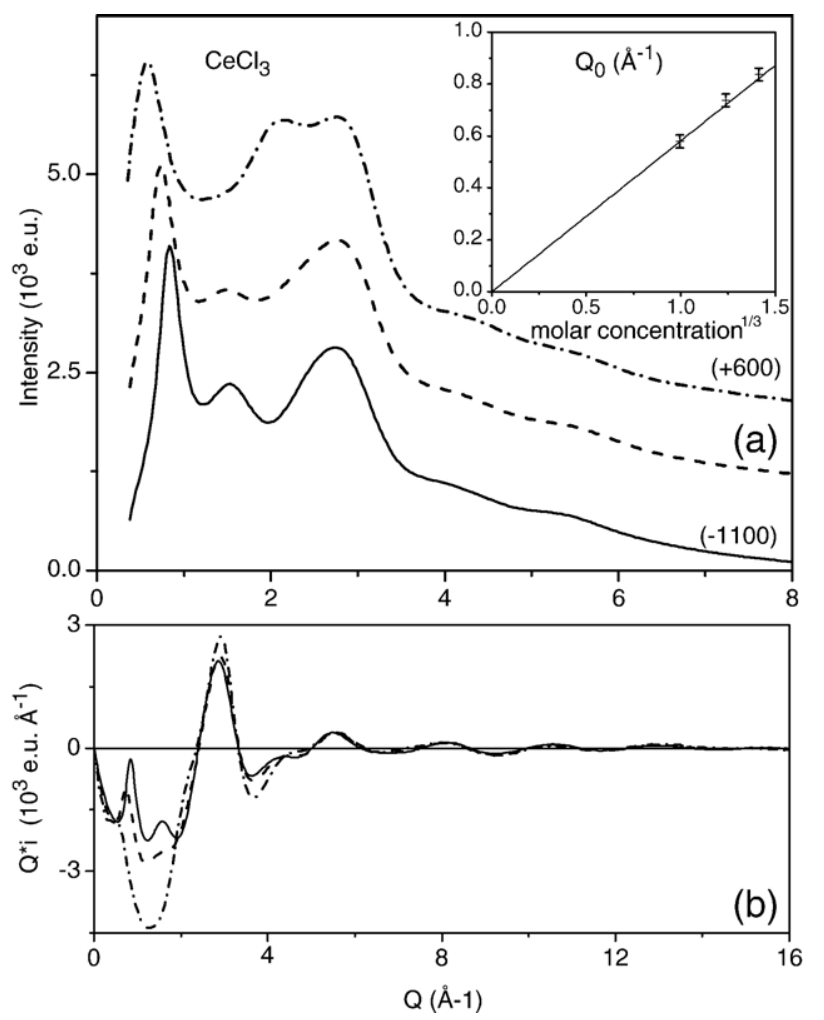

Fig. 8. (a) X-ray diffraction patterns of different concentrated $\mathrm{CeCl}_{3}$ aqueous solutions: (-) $2.81 \mathrm{~mol} \mathrm{dm}^{-3},(--) 1.90 \mathrm{~mol} \mathrm{dm}{ }^{-3}$ and $(-\cdot-) 0.98 \mathrm{~mol} \mathrm{dm}^{-3}$. The variation of the position of the prepeak versus the power $1 / 3$ of the molar concentration is represented (inset). The straight line was calculated considering that the hydrated cations are locally ordered in a face-centered close packing. (b) Reduced intensity of different concentrations of $\mathrm{CeCl}_{3}$ aqueous solutions. 


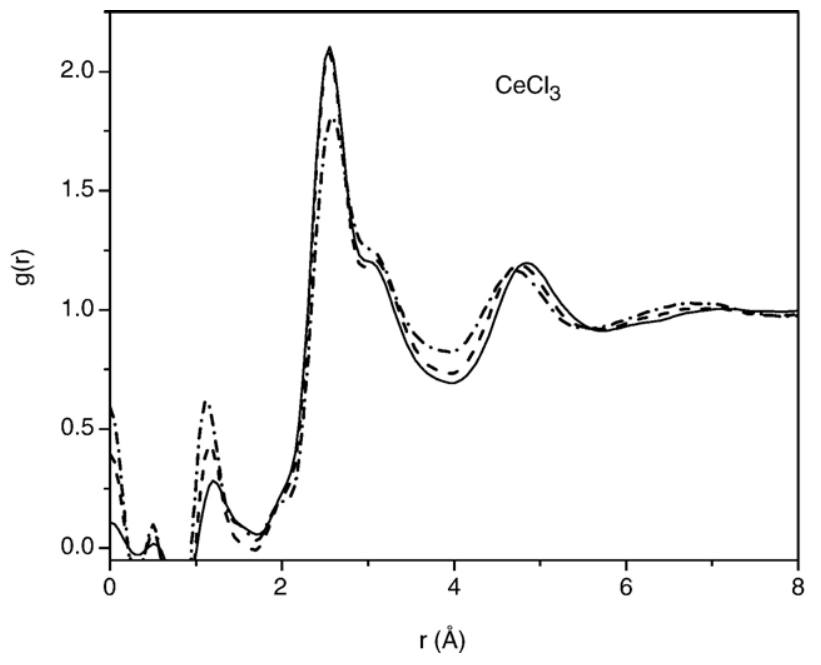

Fig. 9. Pair-correlation function $\mathrm{g}(\mathrm{r})$ of different concentrated $\mathrm{CrCl}_{3}$ aqueous solutions: (-) $2.81 \mathrm{~mol} \mathrm{dm}^{-3},(--) 1.90 \mathrm{~mol} \mathrm{dm}^{-3}$ and $\left({ }^{-} \cdot\right) 0.98 \mathrm{~mol} \mathrm{dm}^{-3}$.

With very symmetrical molecular models similar to those previously suggested for halide solutions in a number of articles already published, the authors were not able to build models appropriate to describe the experimental results (Figs. 3a, b and 4). Difficulties result certainly from the presence of nitrate anions that introduce a relevant lack of symmetry in the interactions between each ion of highest electric charge and its neighbours.

The Raman spectra of these solutions (Fig. 5) are not unambiguously interpreted. Future investigations are desirable. Bands at $\sim 720, \sim 1050, \sim 1340$ and $1420 \mathrm{~cm}^{-1}$ are attributed to internal vibrations of this anion in agreement with what is done with other solutions where the nitrate anion is present. The nitrate anion is assumed as an equilateral triangle with the nitrogen nucleus at the center. The pair $\left(1340,1420 \mathrm{~cm}^{-1}\right)$ corresponds to a destruction of the degeneracy of the vibration $v_{3}$ of the nitrate (Fig. 6) by a neighbour water molecule of the solution $[78,79]$. A polarized band observed at $\sim 520 \mathrm{~cm}^{-1}$ in the spectra of three aqueous solutions of chromium nitrate here presented may be assigned, plausibly, to the vibrational motion
Table 2

Structural data of the molecular model for $2.81 \mathrm{~mol} \mathrm{dm}^{-3} \mathrm{CeCl}_{3}$ aqueous solution

\begin{tabular}{llllr}
\hline Correlations & $r$ & $\Delta r$ & $w$ & $r$ th \\
\hline Cation-cation & 9.42 & 1.2 & 12 & 12.56 \\
Cation-water (h) & 2.55 & 0.10 & 16 & 8.26 \\
& 7.49 & 0.7 & 48 & \\
Water (h)-water (h) & 2.94 & 0.13 & 24 & 6.27 \\
& 4.16 & 0.42 & 24 & \\
& 5.09 & 0.52 & 8 & \\
Cation-anion & 5.26 & 0.52 & 24 & \\
Cation-water (f) & 6.03 & 0.61 & 24 & \\
& 4.71 & 0.26 & 12 & 6.56 \\
& 4.68 & 0.65 & 12 & 8.26 \\
& 4.71 & 0.65 & 8 & \\
\hline
\end{tabular}

Values of the mutual distances $r$, r.m.s. fluctuations $\Delta r$, number of particles $w$, and threshold of the continuum $r$ th for each kind of correlation. Distances are in $\AA$.

(h) and (f) mean respectively hydration water molecules and free water molecules.

that consists of the breathing vibration of the solvation shell of the chromium cation. In approximately the same region $\left(v \sim 435 \mathrm{~cm}^{-1}\right)$ a polarized band is observed in the Raman spectrum of a concentrated aqueous solution $\left(4.84 \mathrm{~mol} \mathrm{dm}^{-3}\right)$ of copper nitrate (Fig. 7). An almost similar interpretation was attributed to this band [9].

For cerium chloride solutions the liquid-type quasi-closepacking arrangement of the cerium cation is also satisfactorily verified, namely by the law of proportionality of $Q_{0}$ to the power $1 / 3$ of the value of the concentration of the salt (inset of Fig. 8a). The profiles observed in the X-ray diffraction patterns (Fig. 8a and b), as well as in the pair-correlation function $g(r)$ (Fig. 9) were compared with the obtained ones from approximate molecular models (Fig. 10a and b). To simulate the molecular distribution of the ions and water molecules in $2.81 \mathrm{~mol} \mathrm{dm}^{-3}$ cerium chloride solution the authors suggest a H8 model with eight water molecules (hydration water) in the vertices of a cube and no anion in the first coordination shell of the cerium cation (discrete distribution). A different model H9 with nine water molecules (and no anion) with a less
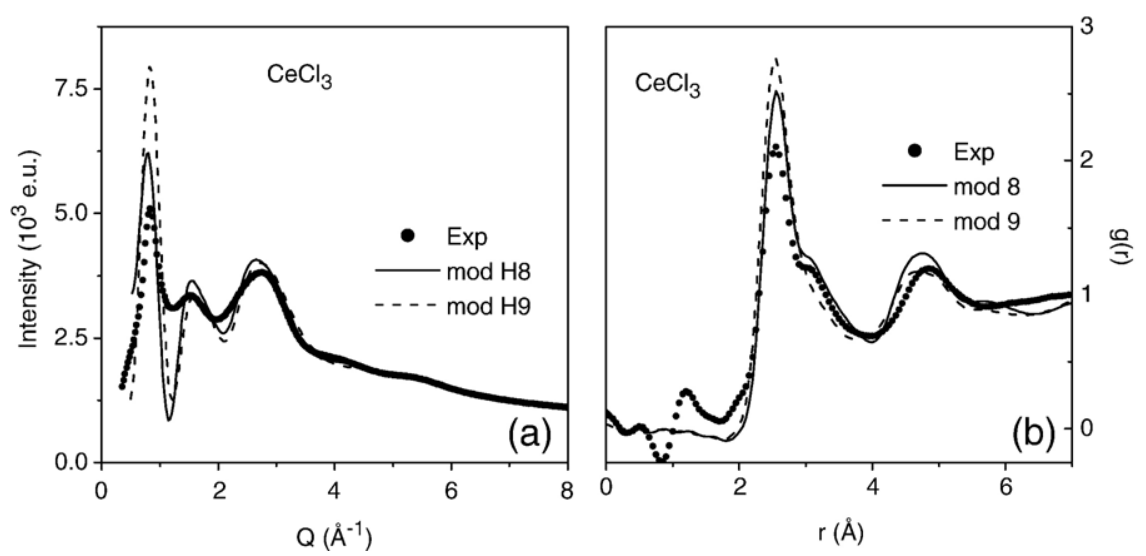

Fig. 10. (a) Experimental $(\cdots)$ and calculated results for $2.81 \mathrm{~mol} \mathrm{dm}^{-3} \mathrm{CeCl}_{3}$ aqueous solution from models $\mathrm{H} 8(-)$ and $\mathrm{H} 9$ (--) (hydrates of the cerium cation with 8 and 9 water molecules, respectively): (a) X-ray diffraction patterns (b) pair-correlation function $g(r)$. 
symmetrical configuration was also tried for the first solvation cation shell. This proposed hydrate with nine water molecules has a shape similar to that one adopted for the lanthanum hydrate previously described [7]. However the agreement with the obtained X-ray diffraction intensity profile was not significantly modified. Anions and water molecules, not included in the first solvation shell of the cerium cation, (free water molecules) are tentatively distributed over the vacant sites of the quasi-close-packing of the hydrated cations. The values of the more relevant mutual distances are given in Table 2.

So, we consider that a first solvation shell of the cerium cation with 8-9 water molecules and no anion appears to be a satisfactory complementary confirmation of the existence of a liquid-type quasi-close-packing arrangement to simulate the internal structure of the cerium chloride solution.

The disagreement of the experimental profile in the reciprocal as well as in the real space with the obtained one for the proposed models results, perhaps, from their excessive symmetry assumed, once more, for requirements of easier calculations. If the distribution of the values of the distances between the ions and between the ions and the water molecules had been more largely spread out than the obtained one from the (here used) models a better agreement with experimental profile should be certainly obtained.

The Raman spectra of three concentrated aqueous solutions of cerium chloride display a weak polarized band close to $330 \mathrm{~cm}^{-1}$ (Fig. 11). Its intensity lowers, apparently, when the concentration of the salt decreases. A band with the same value of its wave number is observed when the Raman spectra of a succession of concentrated aqueous solutions of cerium nitrate are compared (Fig. 12). The behaviour of the succession of the values of its intensity is analogous to that observed for the solutions of cerium chloride. The existence of different cationanion pairs was suggested [35] for concentrated aqueous solutions of cerium nitrate but the presence of these complex structures was not obvious in the course of our studies. The

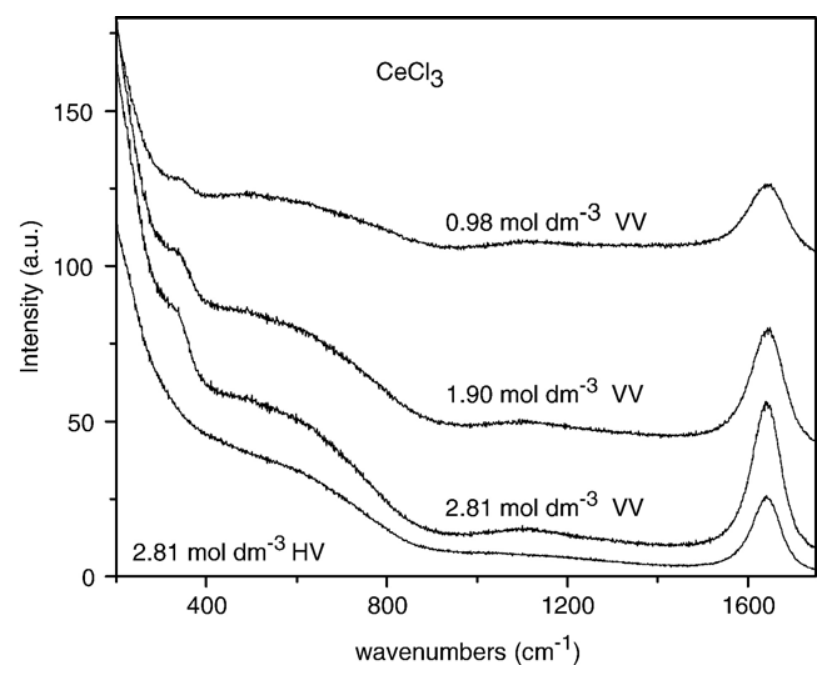

Fig. 11. Polarized $I_{\mathrm{VV}}$ Raman spectra of $0.98,1.90$ and $2.81 \mathrm{~mol} \mathrm{dm}^{-3} \mathrm{CeCl}_{3}$ aqueous solutions. The lower spectrum displays the profile of the depolarized $I_{\mathrm{HV}}$ spectra for the $2.81 \mathrm{~mol} \mathrm{dm}^{-3}$ solution.

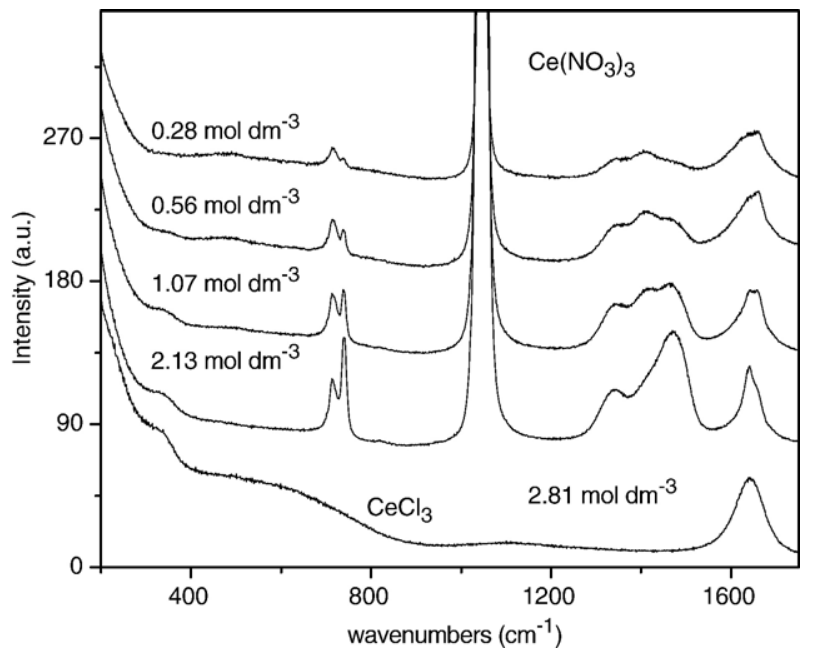

Fig. 12. Polarized $I_{\mathrm{VV}}$ Raman spectra of $0.28,0.56,1.07$ and $2.13 \mathrm{~mol} \mathrm{dm}^{-3}$ cerium nitrate solutions and of $2.81 \mathrm{~mol} \mathrm{dm}^{-3}$ cerium chloride solutions. A band observed in all the spectra at about $330 \mathrm{~cm}^{-1}$; so, it can be tentatively assigned to the breathing vibration of the cerium hydrate.

realisation of more investigations is certainly desirable in order to elaborate a precise conclusion. However it seems that the assignment of this Raman band to the breathing vibration of a cerium hydrate may be suggested with an amount of plausibility.

\section{Conclusion}

The existence of an intermediate range order is considered as very plausible for aqueous solutions of chromium nitrate and for solutions of cerium chloride in the concentration ranges $1.0-2.5 \mathrm{~mol} \mathrm{dm}^{-3}$ and $1-3 \mathrm{~mol} \mathrm{dm}^{-3}$ respectively. The approximate assignment of quasi symmetrical models similar to those used for many solutions analyzed and reported in previous articles [1-13] seems to be acceptable to interpret the X-ray diffraction experiments obtained from concentrated aqueous solutions of cerium chloride. Moreover for cerium chloride solutions Raman experiments are compatible with the obtained ones by X-ray diffraction.

The existence of a liquid-type quasi-close-packing seems to be a universal property of concentrated aqueous solutions of salts constituted by small ions of very different valences.

\section{Acknowledgements}

The authors are pleased to thank Thomas Buslaps and Veijo Honkimaki for their assistance on beamline ID15b during the X-ray diffraction experiments at ESRF. The authors thank JeanLuc Bruneel and David Talaga for their technical assistance during the Raman experiments at the LPCM, Université Bordeaux 1, as well as Maria Clara Carreiro da Costa, who prepared and analyzed the solutions investigated.

We thank 'Fundação Calouste Gulbenkian' and 'Energia de Portugal (EDP)' for having financed the acquisition of some equipment (a powerful $\mathrm{Ar}^{+}$laser) for the experiments. A.M. Gaspar acknowledges the financial support given by 'Fundação para a Ciência e Tecnologia'. 


\section{References}

[1] M. Alves Marques, M.I. de Barros Marques, Proc. K. Ned. Akad. Wet., Ser. B Phys. Sci. 77 (1974) 286.

[2] M.I. de Barros Marques, M.I. Cabaço, M.A. Sousa Oliveira, M. Alves Marques, Chem. Phys. Lett. 91 (1982) 222.

[3] M. Alves Marques, M.I. Cabaço, Chem. Phys. Lett. 123 73-86, 124487 (1986) (E).

[4] M. Alves Marques, M.I. Cabaço, M.A. de Sousa Oliveira, Chem. Phys. Lett. 126 (1986) 551.

[5] M.I. Cabaço, M. Alves Marques, M.I. de Barros Marques, G. BushnellWye, M.M.R. Costa, M.J. de Almeida, L.C. Andrade, J. Phys., Condens. Matter 7 (1995) 7409.

[6] M.I. Cabaço, A.M. Gaspar, C.M. de Morais, M. Alves Marques, J. Phys., Condens. Matter 12 (2000) 2623.

[7] M. Alves Marques, M.I. Cabaço, M.I. de Barros Marques, A.M. Gaspar, C.M. de Morais, J. Phys., Condens. Matter 13 (2001) 4367.

[8] M. Alves Marques, M.I. Cabaço, M.I. de Barros Marques, A.M. Gaspar, J. Phys., Condens. Matter 14 (2002) 7427.

[9] M. Alves Marques, M.I. Cabaço, A.M. Gaspar, M.L. de Almeida, J. Mol. Liq. 110 (2004) 23.

[10] A.M. Gaspar, M. Alves Marques, M.I. Cabaço, M.I. de Barros Marques, T. Buslaps, V. Honkimaki, J. Mol. Liq. 110 (2004) 15.

[11] A.M. Gaspar, M. Alves Marques, M.I. Cabaço, M.I de Barros Marques, A.I. Koleniskov, J. Tomkinson, J.C. Li, J. Phys., Condens. Matter 16 (2004) 6343.

[12] M.I. Cabaço, M. Alves Marques, M.I. de Barros Marques, A.M. Gaspar, de M.L. Almeida, J. Mol. Liq. 117 (2005) 69.

[13] M. Alves Marques, M.I. de Barros Marques, M. Isabel Cabaço, A.M. Gaspar, M.P.M. Marques, A.M. Amado, A.M. Amorim da Costa, J. Molec. Liq. 134 (2007) 142.

[14] J.A. Prins, R. Fonteyne, Physica 2 (1935) 1016; J.A. Prins, R. Fonteyne, J. Chem. Phys. 3 (1935) 72 (gives references); J.A. Prins, R. Fonteyne, H. Petersen, Physica 3 (1936) 147.

[15] A. da Silveira, M. Alves Marques, N. Macias Marques, Compt. Rend. Acad. Sci. 252 (1961) 3983; Mol. Phys. 9 (1965) 271.

[16] M. Alves Marques, M.A. Sousa Oliveira, J. Resina Rodrigues, J. Chem. Soc., Faraday Trans. 86 (1990) 471; 87 (1991) 207 (E).

[17] M. Alves Marques, M.A. Sousa Oliveira, J. Resina Rodrigues, R.M. Cavagnat, J. Devaure, J. Chem. Soc., Faraday 86 (1990) 3883.

[18] M.I. de Barros Marques, P. Lagarde, J. Phys., Condens. Matter 2 (1990) 231.

[19] P. Lagarde, M.I. de Barros Marques, in: E. Balerna, S. Bernieri (Eds.), Conf. Proceed Vol 25A 2nd Europ. Conf. on Progress in X-ray Sinchrotron Radiation Research, SIF, Bolonha, 1990, p. 25.

[20] Ana M. Gaspar, Thesis, Univ. Técnica de Lisboa, (2004) 145.

[21] A.K. Dorosh, A.F. Stryshevskii, Zh. Strukt. Khim. 8 (1967) 3408.

[22] R.A. Howe, W.S. Howells, J.E. Enderby, J. Khim. 8 (1974) 1111.

[23] N.H. March, M.P. Tosi, Phys. Lett. 50A (1974) 224.

[24] J.A. Prins, Proc. K. Ned. Akad. Wet. Ser. B83 (1974) 158.

[25] G.W. Neilson, R.A. Howe, J.E. Enderby, Chem. Phys. Lett. 33 (1975) 284.

[26] J.E. Enderby, Proc. R. Soc. Lond., A 345 (1975) 107.

[27] R. Caminiti, G. Licheri, G. Piccaluga, G. Pinna, J. Chem. Phys. 65 (1976) 3134.

[28] W. Bol, T. Welzen, Chem. Phys. Lett. 49 (1977) 189.

[29] R. Mills, N.H. March, P.V. Giaquinta, M. Parrinello, M.P. Tosí, Chem. Phys. 26 (1977) 237.

[30] R. Caminiti, Magini, Chem. Phys. Lett. 54 (1978) 600.

[31] A. Habenschuss, F.H. Spedding, J. Chem. Phys. 70 (1979) 2797; 73 (1980) 442.

[32] R. Caminiti, G. Licheri, G. Piccaluga, G. Pinna, Rev. Inorg. Chem. 1 (1979) 333.

[33] G. Licheri, G. Piccaluga, G. Pinna, J. Am. Chem. Soc. 101 (1979) 5438.

[34] J.E. Enderby, G.W. Neilson, Adv. Phys. 29 (1980) 323.

[35] R. Caminiti, G. Licheri, G. Paschina, G. Piccaluga, G. Pinna, Z. Naturforsch. 35a (1980) 1361.
[36] A.K. Soper, J.E. Enderby, G.W Neilson, Rep. Prog. Phys. 44 (1981) 595.

[37] R. Caminiti, G. Licheri, G. Paschina, G. Piccaluga, G. Pinna, Z. Naturforsch. 36a (1981) 831.

[38] G. Pálinkás, E. Kalman, Z. Naturforsch. 36a (1981) 1367.

[39] M. Magini, G. Paschina, G. Piccaluga, J. Chem. Phys. 76 (1982) 1116 (and references therein).

[40] G. Pálinkás, T. Radnai, W. Dietz, G.I. Szasz, K. Heinzinger, Z. Naturforsch. 37a (1982) 1049.

[41] J.E. Enderby, The Physics and Chemistry of Aqueous Ionic Solutions, 129, NATO ASI Series C 205, in: M.-C. Bellissent-Funel, G.W. Neilson (Eds.), Reidel, 1987.

[42] G.W. Neilson, J.E. Enderby, Proc. R. Soc. Lond., A 390 (1983) 353 (and references therein).

[43] T. Iijima, K. Nishikawa, Chem. Phys. Lett. 115 (1985) 522.

[44] S.C. Moss, D.L. Price, Physics of Disordered Materials, ed D.Adler, H. Fritzshe and S.R. Ovshinsky (New York Plenum) 1985, p 77.

[45] D.L. Price, S.C. Moss, R. Reijers, M.L. Saboungi, S. Susman, J. Phys., Condens. Matter I (1989) 1005.

[46] W. Meir, P. Bopp, M.M. Probst, E. Spohr, J.-L. Lin, J. Phys. Chem. 94 (1990) 4672.

[47] S.R. Elliot, J. Phys., Condens. Matter 4 (1992) 7661 (and references therein).

[48] M.P. Tosi, D.L. Price, M.L. Saboungi, Annu. Rev. Phys. Chem. 44 (1993) 173.

[49] H. Ohtaki, T. Radnai, Chem. Rev. 93 (1993) 1157.

[50] P.S. Salmon, Proc. R. Soc. Lond., A 445 (1994) 351.

[51] J.C. Wasse, P.S. Salmon, J. Phys., Condens. Matter 11 (1998) 1389.

[52] J.C. Wasse, P.S. Salmon, J. Phys., Condens. Matter 11 (1999) 9293.

[53] M.P. Tosi, J. Mol. Liq. 83 (1999) 23 (and references therein).

[54] Z. Lin, W. Youshi, B. Xiufand, L. Hui, W. Weimin, L. Jingguo, L.Ning, J. Phys., Condens. Matter 11 (1999) 7969 and references therein.

[55] M.C.C. Ribeiro, J. Phys., Condens. Matter 17 (2005) 453.

[56] M.C.C. Ribeiro, Phys. Rev., B 73, (2006), 014201: 1.

[57] J.P. Mathieu, M. Lounsbury, Faraday Soc. 9 (1950) 196.

[58] J.T. Miller, D.E. Irish, Can. J. Chem. 45 (1967) 147.

[59] D.L. Nelson, D.E. Irish, J. Chem. Soc., Faraday 69 (1973) 156.

[60] R. Caminiti, G. Licheri, G. Piccaluga, G. Pinna, Chem. Phys. 19 (1977) 371.

[61] R. Caminiti, G. Licheri, G. Piccaluga, G. Pinna, Chem. Phys. 69 (1978) 1.

[62] R. Caminiti, Chem. Phys. Lett. 86 (1982) 214.

[63] R. Caminiti, P. Cucca, A. D’Andrea, Z. Naturforsch. 38a (1983) 533.

[64] R. Caminiti, G. Cerioni, G. Crisponi, P. Cucca, Z. Naturforsch. 43 (1988) 317.

[65] M.C. Read, M. Sanström, Acta Chem. Scand. 46 (1992) 1177.

[66] R.D. Broadbent, G.J. Neilson, M. Sandström, Phys., Condens. Matter 4 (1992) 639 (and references therein).

[67] A. Fratiello, V. Kubo-Anderson, S. Azimi, E. Marinez, D. Matejka, R. Perrigan, B. Yao, J. Solution Chem. 21 (1992) 651.

[68] P.L. Reis, A. Paez, S.D. Moreno, S. Pattanaik, I. Persson, Sandström, Inorg. Chem. 37 (1998) 6675 (and references therein).

[69] L. Helm, A.E. Merbach, Coord. Chem. Rev. 187 (1999) 151 (and references therein).

[70] C. Kritayakornupong, K. Plankensteiner, B.M. Rode, J. Comput. Chem 25 (2004) 1576.

[71] B.E. Warren, X-Ray Diffraction, Addison-Wesley, M.A. Reading, 1968.

[72] A.J.C. Wilson (Ed.), International Tables for Crystallography C, 1992.

[73] C.T. Chantler, J. Phys. Chem. Ref. Data 29 (2000) 597; J. Phys. Chem Ref. Data 24 (1995) 71.

[74] G. Pálinkás, T. Radnai, Acta Crystallogr., A 32 (1976) 666.

[75] F. Hajdu, Acta Crystallogr., A 28 (1972) 250.

[76] J. Krogh-Moe, Acta Crystallogr. 9 (1956) 951.

[77] N. Norman, Acta Crystallogr. 10 (1957) 370.

[78] D.E. Irish, A.R. Davis, Can. J. Chem. 46 (1968) 943.

[79] R. Caminiti, G. Licheri, G. Piccaluga, G. Pinna, J. Chem. Phys. 68 (1978) 1967. 